\title{
SOME EXACT BERNSTEIN-SZEGŐ INEQUALITIES ON THE STANDARD TRIANGLE
}

\section{LOZKo MileV AND Nikola Naidenov}

Abstract. An actual problem in the theory of approximations is to extend the univariate inequality of Bernstein to the multivariate setting. This question is satisfactorily settled in the case of a centrally symmetric convex body. In spite of the presence of good estimates, exact inequalities of Bernstein's type for nonsymmetric convex bodies are not known.

We prove that the approach based on the Krein-Milman theorem can be applied to maximize the nonlinear functional, which corresponds to the estimate of Bernstein-Szegô type for the gradients of arbitrary polynomials on convex bodies.

As applications we prove exact Bernstein-Szegó inequalities for some classes of bivariate polynomials on the standard triangle $\Delta$. Note that in a certain sense $\Delta$ is the least symmetric convex body in $\mathbb{R}^{2}$.

Mathematics subject classification (2010): 41A17, 26C05, 26B25.

Keywords and phrases: Bernstein-Szegő inequalities, polynomials, extreme points.

\section{REFERENCES}

[1] M. BARAN, Bernstein type theorems for compact sets in $\mathbb{R}^{n}$ revisited, J. Approx. Theory 79 (1994), 190-198.

[2] B. Bojanov, Markov-type Inequalities for Polynomials and Splines, in: "Approximation Theory X: Abstract and Classical Analysis" (C. K. Chui, L. L. Schumaker, J. Stöckler, Eds.), pp. 31-90, Vanderbilt University Press, Nashville, TN, 2002.

[3] P. Borwein And T. ERdÉlyi, Polynomials and Polynomial Inequalities, Graduate Texts in Mathematics, 161, Springer-Verlag, New York, 1995.

[4] A. KRoó AND Sz. Gy. RÉvÉSZ, On Bernstein and Markov-type inequalities for multivariate polynomials on convex bodies, J. Approx. Theory 99 (1999), 134-152.

[5] L. Milev, N. Naidenov And Sz. RÉvÉSz, Estimates for the Derivatives of Polynomials on the Standard Simplex, in: "Jubilee Conference 125 Years Mathematics and Natural Sciences at Sofia University St. Kliment Ohridski”, Book of Abstracts, pp. 53-55, St. Kliment Ohridski University Press, Sofia, 2014 [in Bulgarian].

[6] L. B. Milev AND SZ. Gy. RÉvÉsZ, Bernstein's inequality for multivariate polynomials on the standard simplex, J. Inequal. Appl. 2005:2 (2005), 145-163.

[7] R. T. Rockafellar, Convex Analysis, Princeton University Press, Princeton, New Jersey, 1970.

[8] Y. Sarantopoulos, Bounds on the derivatives of polynomials on Banach spaces, Math. Proc. Camb. Phil. Soc. 110 (1991), 307-312. 